\title{
COMPORTAMENTO ASSINTÓTICO DAS SOLUÇÕES DE UM SISTEMA DE EQUAÇÕES DE EVOLUÇÃO DA HIERARQUIA AKNS
}

\author{
EDSON LUEDERS ${ }^{1}$
}

LUEDERS, E. Comportamento Assintótico das Soluções de um Sistema de Equações de Evolução da Hierarquia AKNS. Semina:Ci. Exatas/Tecnol., Londrina, v. 17, n. 4, p. 365-368, dez. 1996.

RESUMO: Consideramos um sistema de equações de evolução não lineares que possui soluções globais para dados iniciais pequenos. Demonstra-se que as soluções são assintóticas a soluções do problema linear associado quando o tempo tende a infinito. O problema de encontrar soluções não lineares que sejam assintóticas a soluções lineares dadas é investigado.

PALAVRAS-CHAVE: Equações de evolução, soluções globais, hierarquia AKNS.

\section{INTRODUÇÃO}

Consideramos neste artigo o problema de valor inicial

$$
\left\{\begin{array}{l}
i \partial, q=-\frac{1}{2} \Delta q+q^{2} r \\
i \partial, r=\frac{1}{2} \Delta r-q r^{2} \\
q(x, 0)=q_{0}(x) \\
r(x, 0)=r_{0}(x)
\end{array}\right.
$$

onde $q(x, \mathrm{t})$ e $r(x, t)$ são funçöes complexas, $x \in \boldsymbol{R}^{n}$ e $t$ $\geq 0$. O sistema (1.1) pertence à hierarquia AKNS de sistemas hamiltonianos completamente integráveis, e portanto suas soluções podem ser obtidas pelo método de espalhamento inverso (Drazin et al., 1989; Newell, 1985). Nos casos em que $r= \pm q,(1.1)$ se reduz à equação de Schrödinger năo linear:

$$
i \partial_{i} q=-\frac{1}{2} \Delta q \pm||^{2} q \text {. }
$$

Nosso objetivo é estudar o comportamento assintótico das soluções globais de (1.1) utilizando métodos de Análise Matemática. No que se segue denotaremos por $W^{m, p}\left(\mathbf{R}^{n}\right)$ os espaços de Sobolev com norma:

$$
\|u\|_{m, p}=\sum_{\alpha: \leqslant m}\left\|\partial{ }^{\alpha} u\right\|_{\omega, p}
$$

onde $m \in N, I \leq p<\infty \mathrm{e}$

$$
\|u\|_{\phi, p}=\left(\int_{R^{\prime \prime}}|u(x)|^{P} d x\right)^{y_{p}}
$$

Quando $\rho=2$ escrevemos $W^{m, 2}=H^{m}$ e se $m=O$ então $W^{\prime \prime p}=L^{\mu}$. Definimos os espaços $W^{m, s}$ $=\boldsymbol{W}^{m, n} \times W^{m, p}, \boldsymbol{H}^{m}=H^{m} \times H^{m} . O$ sistema $(1.1)$ pode ser reescrito camo

$$
\left\{\begin{array}{l}
i \partial, u=A u+f(u) \\
u(x, 0)=u_{0}(x)
\end{array}\right.
$$

onde:

$$
\begin{gathered}
u=\left(\begin{array}{l}
q \\
r
\end{array}\right), \quad u_{0}=\left(\begin{array}{l}
q_{0} \\
r_{0}
\end{array}\right), \\
A=-\frac{1}{2}\left(\begin{array}{cc}
\Delta & 0 \\
0 & -\Delta
\end{array}\right), \quad f(u)=\left(\begin{array}{c}
q^{2} r \\
-q r^{2}
\end{array}\right)
\end{gathered}
$$


(1.3) Proposição: Se $u_{0} \in H^{m}\left(\boldsymbol{R}^{n}\right), m>n / 2$, então existem $T>0$ e uma única $u \in C\left([0, T] ; \boldsymbol{H}^{m}\right)$ solução de (1.2).

Demonstraçāo: Do fato que $\boldsymbol{H}^{m}\left(\boldsymbol{R}^{n}\right)$ é uma álgebra de Banach se $m>n / 2$ decorre facilmente que $f: H^{m} \rightarrow H^{n}$ é localmente lipschitziana, o que implica a proposição (PAZY, 1985, p.190).

\section{O PROBLEMA GLOBAL}

Nesta seção provaremos que a solução de (1.2) é global se o dado inicial é pequeno e $n \geq 2$.

(2.1) Teorema: Sejam $u_{0} \in H^{m+k}\left(\boldsymbol{R}^{n}\right) \cap W^{m+k} \propto$ ${ }^{s}\left(\boldsymbol{R}^{n}\right), n \geq 2, k, m \in N, k>2 n / 3, m>n / 6, m-k+1>n /$ 6. Existe $\delta>0$ independente de $u_{i j}$ tal que se $\left\|u_{0}\right\|_{m+k .2}<\delta$ e $\left\|u_{0}\right\|_{m+k, 6 / 5}<\delta$ entāo a soluçāo $u(t)$ de (1.2) pode ser estendida a todo $t \geq 0$. Além disso,

$$
\|u(t)\|_{m .6} \leq c(1+t)^{-n / 3}
$$

para todo $t \geq 0$.

Para demonstrar o teorema acima necessitamos das seguintes estimativas (no que se segue, supomos sempre que os índices $m, n, k$ satisfazem as hipóteses do teorema (2.1)):

(2.2) Lema: Sejam $u, v \in H^{n+k}\left(R^{n}\right)$. Então

(a)

$$
\|f(u)\|_{m+k, 6 / 5} \leq c\|u\|_{m, 6}^{2}\|u\|_{m+k, 2}
$$

$$
\begin{aligned}
& \text { (b) }\|f(u)-f(v)\|_{m+k, 2} \leq c\left(\|u\|_{m, 6}^{2}+\|v\|_{m, 6}^{2}\right)\left\|_{u}-v\right\|_{m+\alpha, 2}+ \\
& +c\left(\|u\|_{m+k, 2}+\|v\|_{m+k, 2}\right)\left(\|u\|_{m, 6}+\|v\|_{m, 6}\right)\|u-v\|_{m, 6}
\end{aligned}
$$

Demonstração: Para provar (a) necessitamos estimar

$$
\left\|q^{2} r\right\|_{m+k, 6 / 5}=\sum_{x \leq m+k}\left\|\partial^{\alpha}\left(q^{2} r\right)\right\|_{0,6 / 5} .
$$

Pela regra de Leibniz, $\partial^{\alpha}\left(q^{2} r\right)$ é uma combinaçāo linear de termos da forma $\left(\partial^{\gamma} q\right)\left(\partial^{\eta} q\right)\left(\partial^{{ }} r\right)$ em que os índices $\gamma, \eta, \varsigma \in \mathbf{Z}^{n}$ sastifazem $|\gamma|+|\eta|+|\varsigma| \leq m+k$.

Usando a desigualdade de Hölder

$$
\|f g h\|_{0,6 / 5} \leq c\|f\|_{f, 6}\|g\|_{0,6}\|h\|_{0,2}
$$

de modo que a norma $L^{2}$ seja sempre aplicada a um fator com indice maior que $m$ obtemos $\left\|q^{2} r\right\|_{m+k, 6 / 5} \leq c\|u\|_{m, 6}^{2}\|u\|_{m+k, 2}$.

Para provar (b) procedemos de modo análogo e utilizamos as imersões de Sobolev $H^{m+k} \subset W^{m .0} \subset$ $W^{k \cdot 1}: \infty$. $t \in \mathbf{R}$

(2.3) Lema: Se $u_{0} \in \mathbf{W}^{\mathrm{m}+\text {.6/s }}$ entāo para todo

$$
\left\|e^{-z+A} u_{0}\right\|_{m, 6} \leq c(1+|t|)^{-n / 3}\left\|u_{0}\right\|_{m+k, 6 / 5}
$$

Demonstraçāo: (Sulem et al., 1986, proposição 4.1 ).

Demonstração do Teorema (2.1): É suficiente provar que a solução $u(t)$ permanece uniformemente limitada em qualquer intervalo onde está definida. O método da demonstração é baseado em (Sulem et al., 1986).

A solução $u(t)$ satisfaz a equação integral (Pazy, 1983, p.185):

(2.4) $u(t)=e^{-i t A} u_{0}-i \int_{0}^{t} e^{-i(t-s) A} f(u(s)) d s$.

Como $e^{i t A}$ é uma isometria em $\boldsymbol{H}^{m+k}$, segue de (2.4) e do lema (2.2) que

$$
\|u(t)\|_{m+k, 2} \leq\left\|u_{0}\right\|_{m+k .2}+c \int_{0}^{r}\|u(s)\|_{m, 6}^{2}\|u(s)\|_{m+k, 2} d s
$$


a desigualdade de Gronwall implica que

(2.5) $\|u(t)\|_{m+k, 2} \leq\left\|u_{0}\right\|_{m+k, 2} \exp \left(c \int_{0}^{t}\|u(s)\|_{m, 6}^{2} d s\right)$.

Ainda de (2.4) temos, com a ajuda dos lemas (2.2) e (2.3),

(2.6)

$$
\begin{gathered}
\|u(t)\|_{m, 6} \leq c(1+t)^{-m / 3}\left\|u_{0}\right\|_{m+k, 6 / 5}+ \\
+c \int_{0}^{t}(1+t-s)^{-n / 3}\|u(s)\|_{m, 6}^{2}\|u(s)\|_{m+k, 2} d s .
\end{gathered}
$$

Defina

$$
M(T)=\sup _{0 \leq t \leq T}(1+t)^{n / 2}\|u(t)\|_{m, 6} .
$$

As desigualdades (2.5) e (2.6) podem ser reescritas, respectivamente, como

$$
\begin{aligned}
\|u(t)\|_{m+k, 2} & \leq\left\|u_{0}\right\|_{m \rightarrow k, 2} \exp \left[c M(T)^{2} \int_{n}^{t}(1+s)^{-2 n / 3} d s\right] \\
& \leq\left\|u_{0}\right\|_{m+k .2} e^{c M(T)^{2}}
\end{aligned}
$$

(2.8)

$$
\begin{aligned}
& M(T) \leq c\left\|u_{0}\right\|_{m+k, 6 / 5}+ \\
& +c\left\|u_{0}\right\|_{m+k, 2} M(T)^{2} e^{r^{\prime} M(T)^{2}} \sup _{n \leq t \leq T} \int_{0}^{t} \frac{(1+t)^{n / 3}}{(1+t-s)^{m+3}(1+s)^{2 n / 3}} d s \\
& \leq c\left\|\boldsymbol{u}_{\mathrm{o}}\right\|_{m+\cdot k, 6 / 5}+c^{\prime \prime}\left\|\boldsymbol{u}_{0}\right\|_{m-k, 2} M(T)^{2} e^{\left(x^{*} M(T)^{2}\right.} .
\end{aligned}
$$

(Note que as integrais em (2.7) e (2.8) são uniformemente limitadas pois $n \geq 2$.)

Designando max $\left\{c, c_{,}, c^{\prime \prime}\right\}$ por $c$ temos de (2.8)

$$
M(T) \leq c \delta+c \delta M(T)^{2} e^{i M(T)^{2}}
$$

Defina a função $f(x)=c \delta\left(1+x^{2} e^{c x^{2}}\right)-x$, que é convexa e tem exatamente duas raizes $0<K_{\text {, }}$ $<K_{z}$, se $\delta>0$ é suficienternente pequeno. Observe que $f(x)$ é negativa no intervalo $\left(K_{1}, K_{2}\right)$ e positiva se $x<K$, ou $x>K_{2}$. Temos $f(c \delta)>0$ e $f^{\prime}(c \delta)<0$, portanto $0<c \delta<K_{\text {, }}$. Além disso,

$$
M(0)=\left\|u_{0}\right\|_{m, 6} \leq c^{\prime}\left\|u_{0}\right\|_{m+k .2}<c^{\prime} \delta
$$

pela desigualdade de Sobolev. Denotando $\max \left(c, c^{\prime}\right)$ novamente por $c$ segue que $M(O)<c \delta<K_{\text {, }}$. Como $f(M(T)) \geq 0$ por (2.9) e $M(T)$ depende continuamente de $T$, concluímos que $M(T) \leq K_{1}$ para todo $T$. $O$ teorema está, portanto, demonstrado.

\section{COMPORTAMENTO ASSINTÓTICO DAS SOLUÇŌES}

Provaremos nesta seção que a solução de (1.2) é assintótica a uma soluçāo do problema linear

(3.1) Proposição: Se $u_{0} \in \boldsymbol{H}^{m+k} \cap \boldsymbol{W}^{m+k, 6 / 3}$ satisfaz as hipóteses do teorema (3.1) entäo existe $u_{+}$ $\in \boldsymbol{H}^{m+k}$ tal que

$$
\lim _{t \rightarrow+\infty}\left\|u(t)-e^{-i t A} u_{+}\right\|_{m+k, 2}=0
$$

onde $u(t)$ é a solução de (1.2) dada pelo teorema (2.1).

\section{Demonstração: Defina}

$$
u_{+}=u_{0}-i \int_{0}^{+\infty} e^{i s A} f(u(s)) d s
$$

Temos

$$
e^{-i I A} u_{+}=e^{-i m} u_{0}-i \int_{0}^{+\infty} e^{-i(t-s) \wedge} f(u(s)) d s,
$$

que subtraímos de (2.4) para obter

$$
u(t)-e^{-i t A} u_{+}=i \int_{i}^{+\infty} e^{-i(t-s) A} f(u(s)) d s
$$

De (2.1) e (2.2) segue que

$$
\begin{gathered}
\left\|u(t)-e^{-i t A} u_{+}\right\|_{m+k .2} \leq c \int_{t}^{+\infty}\|u(s)\|_{m, 6}^{2}\|u(s)\|_{m+k .2} d s: \\
\leq C \int_{i}^{+\infty}(1+s)^{-2 n / 3} d s \rightarrow 0
\end{gathered}
$$

quando $t \rightarrow+\infty$, o que demonstra a proposição. 
Consideremos agora o problema reciproco, ou seja, dado $u_{+} \in \boldsymbol{H}^{n+k}$ queremos obter uma soluçāo $u(t)$ de (1.2) satisfazendo (3.2).

(3.3) Proposição: Dado $a_{+} \in H^{m+k}\left(\mathbf{R}^{n}\right) \cap W^{m+k}$ $\sigma / S\left(\mathbf{R}^{n}\right), n \geq 4$, existem $T>0$ e uma única $u \in C\left(\left[T_{*}+\infty\right)\right.$, $\left.H^{m+k}\left(R^{n}\right)\right)$ solução de (1.2) satisfazendo

(i) $\sup _{t \geq T}\|\mu(t)\|_{m+k, 2}<\infty$;

(ii) $\sup _{t \geq T}(1+t)^{n / 3}\|u(t)\|_{m, 6}<\infty$;

(iii) $\lim _{t \rightarrow+\infty} \mid u_{t}(t)-e^{-i z A} u_{+} \|_{m+k, 2}=0$.

Demonstraçăo: Dados $r>0, K>0$ e $M>0$, seja $\Lambda$ o conjunto das aplicações $u \in C\left(\left[T_{x}+\infty\right), \boldsymbol{H}^{n+k}\right)$ tais que para todo $t \geq T$

$$
\begin{aligned}
& \left\|u(t)-e^{-i t A} u_{+}\right\|_{m+k, 2} \leq K \\
& \left\|u(t)-e^{-i t A} u_{+}\right\|_{m, 6} \leq M(1+t)^{-n / 3} .
\end{aligned}
$$

Com a métrica

$$
d(u, v)=\sup _{r \geq T}\|u(t)-v(t)\|_{m+k, 2}
$$

o conjunto $\Lambda$ é um espaço métrico completo.
Para $u \in \Lambda$ defina

$$
F(u)(t)=e^{-i t A} u_{+}+i \int_{t}^{+\infty} e^{-i(t-x) A} f(u(s)) d s .
$$

Com o auxílio dos lemas (2.2) e (2.3) mostrase que $F: \Lambda \rightarrow \Lambda$ é uma contraçāo se $T>0$ é suficientemente grande. Pelo teorema do ponto fixo de Banach existe uma única $u \in \Lambda$ tal que

$F(u)(t)=u(t)=e^{-i t A} u_{+}+i \int_{t}^{+\infty} e^{-i(t-s) A} f(u(s)) d s$.

Como na proposição (3.1) prova-se que $u(t)$ satisfaz (3.2). Observe que $u(t)$ é solução do problema nạo linear, pois de (3.4) temos

$$
\begin{aligned}
\partial_{t} u & =-i A e^{-i t A} u_{+}+i \int_{1}^{+\infty}(-i) A e^{-i t-s i A} f(u(s)) d s-i f(u(t))= \\
& =-i A u(t)-i f(u(t)) .
\end{aligned}
$$

Se $w \in C\left([T,+\infty), H^{n+k}\right)$ é outra soluçăo satisfazendo (i),(ii) e (iii), então we $\Lambda$ para uma escolha conveniente das constantes $K$ e $M$. Pelo teorema do ponto fixo de Banach, $u=u, \theta$ a proposição está demonstrada.

LUEDERS, E. Asymptotic behaviour of solutions of am evolution equations system in the AKNS hierarchy. Semina:Ci. Exatas/Tecnológicas, Londrina, v. 17, n. 4, p. 365-368, Dec. 1996.

ABSTRACT: A system of nonlinear evolution equations with global solutions for small data is considered. We prove that the solutions are asymptotic to solutions of the linear problem whem the time goes to infinity. Moreover, we investigate the problem of finding nonlinear solutions asymptotic to given linear solutions.

KEY WORDS: Evolution equations, global solutions, AKNS hierarchy.

\section{REFERÊNCIAS BIBLIOGRÁFICAS}

DRAZIN, P.G., JOHNSON, R.S. Solitons: an Introduction. [S.I] Cambriage University Press, 1989.

NEWELL, A.C. Solitons in Mathematics and Physics Philadelphia, Pa: SIAM.1985.
PAZY, A. Somigroups of Lincar Opcrators and Applications to Partial Diferential Equations. [S.I]: Springer-Verlag. 1983.

SULEM, P.L., SULEM, C., BARDOS, C. On the Continuous Limit for a System of Classical Spins. Commun. Math. Phys., v. 107, p.431-454, 1986 . 